\title{
Mobile Ad-Hoc Networks Applications and Its Challenges
}

\author{
Naeem Raza1, Muhammad Umar Aftab ${ }^{1}$, Muhammad Qasim Akbar², \\ Omair Ashraf', Muhammad Irfan ${ }^{4}$ \\ ${ }^{1}$ Department of Computer Science and IT, University of Sargodha Lyallpur Campus, Faisalabad, Pakistan \\ ${ }^{2}$ Department of Computer Science, GC University, Faisalabad, Pakistan \\ ${ }^{3}$ Department of Computer Science, NFC-IEFR, Faisalabad, Pakistan \\ ${ }^{4}$ Land Record Management and Information System, Faisalabad, Pakistan \\ Email: Naeemraza_lucky@yahoo.com,ms.umaraftab@yahoo.com,emqa1408@gmail.com, \\ omairashraf@outlook.com, multi_com51@hotmail.com
}

Received 18 May 2016; accepted 17 July 2016; published 20 July 2016

Copyright (C) 2016 by authors and Scientific Research Publishing Inc.

This work is licensed under the Creative Commons Attribution International License (CC BY). http://creativecommons.org/licenses/by/4.0/

(c) (7) Open Access

\begin{abstract}
Mobile Ad-Hoc Network (MANET) is an infrastructure less wireless network of autonomous collection of mobile nodes (Smart phones, Laptops, iPads, PDAs etc.). Network is self-configured to reconstruct its topology and routing table information for the exchange of data packets on the joining and leaving of each node on ad-hoc basis. This paper is based on the MANET applications and challenges. The researchers can get the overall concept of MANET as well as its applications and challenges.
\end{abstract}

Keywords

MANET, MANET Challenges and Applications

\section{Introduction}

Many new applications are resulted from progress in the internet discipline because of wireless network technologies. For research and development of wireless network, one of the most auspicious arenas is Mobile AdHoc Network (MANET).

Wireless ad-hoc network is becoming one of the most animated and dynamic field of communication and networks because of fame of movable device and wireless networks that has increased significantly in recent years. A mobile ad-hoc network is formed by collecting portable devices like laptops, smart phones, sensors, etc. that communicate through wireless links with one another. These devices collaborate with each other to offer the 
essential network functions in the nonappearance of immovable organization in a distributed manner. This type of network creates the way for various innovative and stimulating applications by functioning as an independent network or with multiple points of connection to cellular networks or the Internet [1].

Routing of packets to destination is done by the cooperation of nodes of a MANET. The sending and receiving devices may be situated at a much higher distance as compared to transmission radius R, however, each network node can communicate only with nodes placed within its broadcast radius R. All the nodes in a multihop wireless ad-hoc network collaborate with one another to create a network in the absence of infrastructure such as access point or base station.

In order to permit transmission among devices beyond the transmission range in MANET, the mobile devices require advancing data-packets for one another. The network devices can move freely and autonomously in any route. The nodes can detach and attach to the network haphazardly. Thus variations in link states of the node with other nodes are experienced by a node regularly. Challenges for routing protocols operating in MANET are eventually increased the movement in the ad-hoc network, changes in link states and other characteristics of wireless transmission such as attenuation, multipath propagation, interference etc. The challenges are boosted by the numerous sorts of nodes of restricted processing power and competences that may join the network [2].

Ultimate aim and objective of this research work is to analytically review routing protocols of MANET, simulate DSR, TORA and OLSR routing protocols by using simulator and compare the results under different scenarios like with Nodes Density of 25, 50 and 75 nodes, evaluate and analyze these routing protocols under the various environments by using some parameters like WLAN delay, WLAN throughput, WLAN network load, FTP traffic sent and received both by the nodes and server, routing traffic sent and received.

\section{Mobile Ad-Hoc Network}

MANET is basically an organization less network of transportable devices having wireless communication capabilities that can join together at any time and at any place dynamically. In this type of network mobile hosts, sometimes, simultaneously acting as a router, are connected to one another by wireless links and they can easily move randomly hence network topology dynamically change so this makes an autonomous system of mobile nodes having no base station. In MANET each node has limited transmission range so packets are forwarded from any initiating node to any end point node in a network with the help of multiple hopes [3].

\section{Wireless Networks}

Wireless networks not only offer connection flexibility among users at different places they also help in the extension of the network to any building or area without a physical-wired connection. Such connections are of two types; Substructure networks and Ad-Hoc networks as shown in Figure 1.

Access Point (AP), in Infrastructure wireless networks, denotes a central controller for each device. The network can be joined through access point by any node. In order to make the route ready when it is needed, the access point arranges the linking among the Basic Set Services (BSSs). Still, there is a disadvantage of using an organization of network that is the big overhead of preserving the routing tables [4].

Structure less or Ad-Hoc networks lack firm topology or a central controlling point, that's why, transfer and receipt of data-packets is more complex as compared to structured networks.

Wireless networks can be classified as single hop and multi hop as well as infrastructure based and ad hoc based. In single hop wireless networks base station (BS) and wireless devices communicated directly with electromagnetic waves to each other. In multi hop wireless networks wireless devices indirectly communicate with the base station by sending data from one device to other and so on.

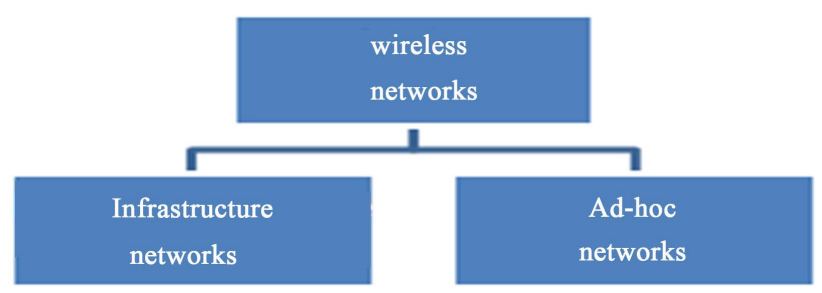

Figure 1. Classifications of wireless networks. 


\subsection{Characteristics of MANET}

Autonomous and infrastructure-less: MANET is independent of conventional structure or central management. Each device plays the role of an independent router and generates independent data because it functions in dispersed P2P style. Fault detection and management becomes difficult as network administration has to be scattered crosswise various nodes.

Multi-hop routing: Every node plays the role of router and forward packets for information distribution among portable hosts. No default router is available.

Dynamic topologies: Because of arbitrary movement of nodes, the network topography (which is classically multi-hop) changes regularly and randomly. This results in changes in routes, common network sub divisions, and perhaps data-packet losses.

Unconventionality in link and node abilities: It is possible for every device to be armed with one or more radio interfaces with each one having changing sending/receiving competences. These may also function transversely various frequency groups. Irregular links may be resulted due to these heterogeneous node radio abilities. In addition to this, processing capability may also vary due to different software/hardware configuration of mobile nodes. Scheming network protocols and algorithms for this diverse network can be complicated, demanding dynamic adjustments to the altering situations (power and channel conditions, traffic load/distribution variations, congestion, etc.). Energy controlled process. Each movable device having restricted battery power supply, processing power is affected. As a result facilities and applications provided by every device are also limited. Additional energy is required because every node has to act as system and router. Additional power is required for packer forwarding to other nodes. So this develops to be a greater problem in mobile ad hoc networks.

Network scalability: Scalability is serious issue to the successful implementation of these networks. Currently, popular network management algorithms could only support static or comparatively minor wireless networks. Numerous mobile ad hoc network applications include large networks huge number of nodes, as found for instance, in sensor networks and tactical networks. The implementation of such a network presents several issues that are needed to be resolved. These challenges include: addressing, routing, location management, configuration management, interoperability, security, high capacity wireless technologies, etc.

\subsection{Benefits of MANET}

- Highly suitable network in such circumstances where fixed infrastructure is too much costly, untrustworthy, not trusted and due to unavailability of such a network.

- Quickly installation with least possible user intervention.

- Detailed planning and installation of base stations is not required.

- Ad hoc networks can be attached to the WWW or Internet, thereby incorporating many different devices and making possible for other users to use available services.

- Capacity, range and energy arguments promote their use in tandem with existing cellular infrastructures as they can extend coverage and interconnectivity.

- MANET also fitted to use the future 4G architecture and their services, aims to provide ubiquitous computer environments that support users in completing their tasks, accessing information and communicating anywhere, anytime and from any device [5].

\section{MANET Environment Variations}

The different MANET setting differences are programmed taking its dynamic topology in to consideration.

In MANETs all the nodes have duplicate abilities and responsibilities, which are labeled as symmetric environment. MANET containing wireless mobile nodes that interconnect without integrated control or recognized structure. These nodes are within each other's radio range can interconnect directly, while expanse nodes depend on their adjoining nodes to forward the packets. In MANETs these node can be a router or a host. In MANET environment, these nodes are allowed to leave or join the system at any time, ensuring a highly energetic network environment paralleled to supported network.

The Irregular Competences in MANETs comprise transmission series and radios series which may change. Speed of movement, battery life and processing capacity will be dissimilar at different nodes. Irregular Respon- 
sibilities contain that some of the nodes may track packets in the network or some of the nodes may perform as leaders for the neighboring nodes such as cluster head. Traffic features may differ in diverse ad hoc networks like timeliness constraints, bit rate reliability necessities, unicast, multicast or geocast, content-based addressing or host-based addressing or capability-based addressing. MANETs may co-operate and also co-exist with a setup based network. Mobility arrangements may be different as people sitting at any airport lounge, taxi cabins, military actions and private area networks. The action of a mobile ad hoc network is reliant on the node mobility design as well as data traffic patterns, topology and radio intervention. Mobility features including speed, direction of movement, predictability, design of movement, consistency of mobility features among different nodes [6].

\section{MANET Challenges}

The subsequent list of issues indicates the inadequacies and restrictions that have to be overwhelmed in a MANET environment:

Restricted wireless transmission range: The radio group will be restricted in the wireless networks and as a result data amounts it can provide much slighter than what a bound network can provide. This involves routing procedures of wireless networks must be use bandwidth in ideal way. This can be achieved through protecting the overhead as minimum as conceivable. The restricted transmission range also enforces restraint on routing procedures for sustaining the topographical information. Particularly in MANETs because of regular variations in topology, preserving the topological data for every node includes more controller overhead which results in additional bandwidth depletion.

Time-varying wireless link characteristics: Wireless channel is liable to a range of broadcast disorders such as path harm, declining, intervention and obstruction. These features resist the series, data rate, and consistency of these cordless transmissions. The range of which these features disturb the transmission that rest on atmospheric situations and flexibility of receiver and transmitter. Even two dissimilar key restraints, Nyquist's and Shannon's theorems that rule over capability to communicate the information at diverse data degrees can be measured.

Broadcast nature of the wireless medium: The broadcast nature of the radio channel, such as transmissions prepared by a device is established by all devices that are in its straight transmission covering area. When a device receives data, no other device in its neighborhood, apart from the sender, must transfer. A device can acquire access to the mutual medium when its communications cannot disturb any constant session. Meanwhile several devices may resist for medium contemporarily, chance of data-packet crashes is very tall in wireless networks. Even the network is liable to concealed terminal issue and transmits storms. Concealed terminal issue mentions to the smash of data-packets at a receipt device because of immediate transmission of the nodes which are outside the straight communication series of the transmitter, but are inside the communication series of the receiver.

Packet losses due to transmission errors: Ad hoc wireless networks practices very advanced packet damage due to reasons such as extraordinary bit error rate (BER) in the wireless channel, enlarged crashes because of the existence of unseen terminals, occurrence of interventions, position reliant controversy, single directional associations, regular pathway breakages due to device movements, and the integral declining characteristics of the wireless passage.

Mobility-induced route changes: The system topography in ad hoc wireless network is extremely active because of node movement; as a result, a constant meeting undergoes numerous pathway breakages. Such position often results in regular path alterations. So flexibility administration is massive investigation theme in ad hoc networks.

Mobility-induced packet losses: Communication contacts in an ad hoc network are insecure such that consecutively conservative procedures for MANETs over a great damage frequency will suffer from performance deprivation. Though, with large frequency of inaccuracy, it is problematic to supply a data-packet to its target.

Battery constraints: It is due to restricted resources that arrange main limitation on the mobile devices in an ad hoc network. Nodes which are contained in such network have restrictions on the supremacy foundation in order to preserve movability, dimension and capacity of the node. Due to accumulation of power and the processing capacity make the nodes heavyweight and less portable. Consequently only MANET devices have to use this resource.

Potentially frequent network partitions: Casually stirring nodes in an ad-hoc network may result in net- 
work panels. Certain cases involve middle nodes to be extremely effected by such separation.

Ease of snooping on wireless transmissions (security issues): Wireless passage being employed for ad hoc networks transmitted in natural surroundings. It is also shared by all devices in the network. Transmission of data through a device is acknowledged by all devices inside straight communication series. So invader is certain to sneak data/information which is communicated within network. The conditions of secrecy could be disrupted if enemy is capable in inferring data assembled by snooping [6].

Routing: In MANETs routing is an important challenge for the performance degradation due to unicasting, multicasting and geocasting demands by the network nodes in contrast to single hope wireless networks. It's because of rapid change in network topology and with different mobility speeds.

Quality of Service: In MANETs quality of service is an important challenge for the differed kind of quality level demands by the network nodes. Its becomes very difficult to fulfill the different levels or priority demands related to quality of service so these network required best control of QoS specially in case of multimedia [7].

Security: In MANET, security is one the important challenge due to its wireless environment. The data of users from one node to another node must be transferred safely and completely. The least privilege principle can also enhance the security of MANET systems as proposed for organizations. Moreover, there are hybrid models are also available that are offering benefits of two access control models with implementations [8] [9].

\section{Applications of MANET}

Some distinctive MANET applications include:

Military field: Ad-Hoc networking can permit army to exploit benefit of conventional network expertise for preserving any info network among vehicles, armed forces, and headquarters of information.

Cooperative work: To facilitate the commercial settings, necessity for concerted computing is very significant external to office atmosphere and surroundings as compared to inner environment. People want getting outside meetings for exchanging the information plus cooperating with each other regarding any assigned task.

Confined level: Ad-Hoc networks are able to freely associate with immediate, in addition momentary hypermedia network by means of laptop computers for sharing the info with all the contestants' e.g. classroom and conference. Additional valid and confined level application may be in domestic network where these devices can interconnect straight in exchanging the information.

PAN and Bluetooth: A PAN is localized and tiny range network whose devices are generally belong to a specified individual. Limited-range MANET such as Bluetooth can make simpler the exchange among several portable devices like a laptop, and a cell phone.

Business Sector: Ad-hoc network could be used for rescuing and emergency processes for adversity assistance struggles, for instance, in flood, fire or earthquake. Emergency saving procedures should take place where damaged and non-existing transmissions structure and quick preparation of a transmission network is required [10].

Sensor Networks: managing home appliances with MANETs in both the case like nearby and distantly. Tracking of objects like creatures. Weather sensing related activities.

Backup Services: liberation operations, tragedy recovery, diagnosis or status or record handing in hospitals, replacement of stationary infrastructure.

Educational sector: arrangement of communications facilities for computer-generated conference rooms or classrooms or laboratories [10].

\section{Conclusion}

MANET is the emerging technology but it has some challenges that must be covered for efficient results. The security is the main challenges in the networks and especially in the wireless technologies such as MANET. We can get better results from MANET by using its applications. The security can be enhanced with the implementation of some security mechanisms.

\section{References}

[1] Raja, M.L. and Baboo, C.D.S.S. (2014) An Overview of MANET: Applications, Attacks and Challenges.

[2] Chitkara, M. and Ahmad, M.W. (2014) Review on MANET: Characteristics, Challenges, Imperatives and Routing 
Protocols. International Journal of Computer Science and Mobile Computing, 3, 432-437.

[3] Mohammad, S., Alsanabani, M. and Alahdal, T. (2014) Comparison Study of Routing Protocols in MANET. International Journal of Ad Hoc, Vehicular and Sensor Networks, 1, 1-9.

[4] Odeh, A., Abdel Fattah, E. and Alshowkan, M. (2012) Performance Evaluation of AODV and DSR Routing Protocols in MANET Networks.

[5] Verma, S. and Singh, P. (2014) Energy Efficient Routing in MANET: A Survey. International Journal of Engineering and Computer Science, 3, 3971-3977.

[6] Mamatha, G. and Sharma, D.S. (2010) Analyzing the MANET Variations, Challenges, Capacity and Protocol Issues. International Journal of Computer Science \& Engineering Survey, 1, 14-21. http://dx.doi.org/10.5121/ijcses.2010.1102

[7] Goyal, P., Parmar, V. and Rishi, R. (2011) Manet: Vulnerabilities, Challenges, Attacks, Application. International Journal of Computational Engineering \& Management, 11, 32-37.

[8] Aftab, M.U., Nisar, A., Asif, D., Ashraf, A. and Gill, B. (2013) RBAC Architecture Design Issues in Institutions Collaborative Environment. International Journal of Computer Science Issues, 10, 216-221.

[9] Aftab, M.U., Habib, M.A., Mehmood, N., Aslam, M. and Irfan, M. (2015) Attributed Role Based Access Control Model. Conference on Information Assurance and Cyber Security (CIACS), Rawalpindi, 18 December 2015, 83-89. http://dx.doi.org/10.1109/CIACS.2015.7395571

[10] Chitkara, M. and Ahmad, M.W. (2014) Review on MANET: Characteristics, Challenges, Imperatives and Routing Protocols. International Journal of Computer Science and Mobile Computing, 3, 432-437.

\section{Submit or recommend next manuscript to SCIRP and we will provide best service for you:}

Accepting pre-submission inquiries through Email, Facebook, Linkedin, Twitter, etc A wide selection of journals (inclusive of 9 subjects, more than 200 journals)

Providing a 24-hour high-quality service

User-friendly online submission system

Fair and swift peer-review system

Efficient typesetting and proofreading procedure

Display of the result of downloads and visits, as well as the number of cited articles

Maximum dissemination of your research work

Submit your manuscript at: http://papersubmission.scirp.org/ 